Supporting information to

\title{
Single particle spectroscopic investigation on the interaction between exciton transition of cyanine dye J-aggregates and localized surface plasmon polarization of gold nanoparticles
}

\author{
Takayuki Uwada, Ryo Toyota, Hiroshi Masuhara, and Tsuyoshi Asahi* \\ Department of Applied Physics, Osaka University, Yamadaoka 2-1, Suita, Osaka \\ 565-0871, Japan
}

Corresponding Author: E-mail: asahi@ap.eng.osaka-u.ac.jp

Numerical calculations of light scattering spectra of gold core coated with J-aggregate on the basis of the Mie theory using the algorithm described in the text (C. F. Bohren and D. R. Huffman, Absorption and Scattering of Light by Small Particles (Wiley, New York, 1983)). In the calculation, we use 1.4 as the refractive index of medium and the refractive index of bulk gold as complex refractive index of the particle. The complex refractive index of the PIC J-aggregate layer is given in Figure 3(c) in text. 


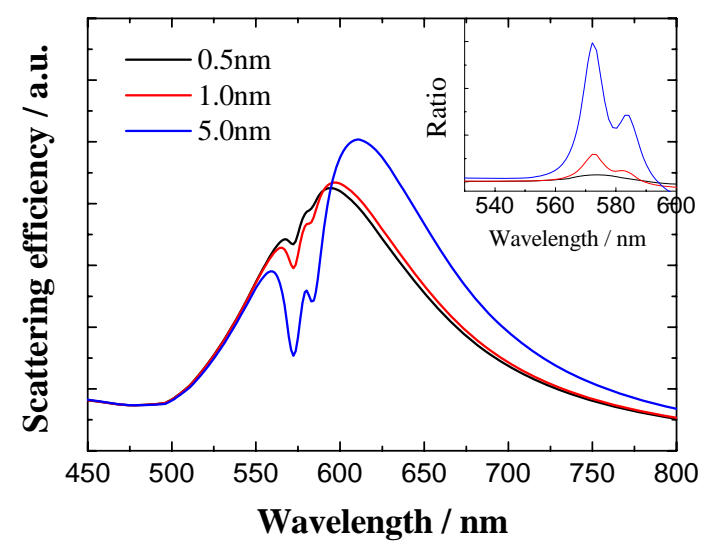

Figure S1. Calculated scattering spectra of a gold nanoparticle (100 nm diameter) coated with PIC J-aggregate layer of $0.5 \mathrm{~nm}$ (black), $1.0 \mathrm{~nm}$ (red), and $5.0 \mathrm{~nm}$ (blue) thickness, respectively, in a medium of the refractive index of 1.4 on the basis of the Mie theory using a metal core-J-aggregate shell model in Figure 3. Insertion shows the spectral ratio calculated by dividing the spectra of the J-aggregate coated gold nanoparticle with that of the naked nanoparticle. The dip position does not depend on the layer thickness in the calculation. 

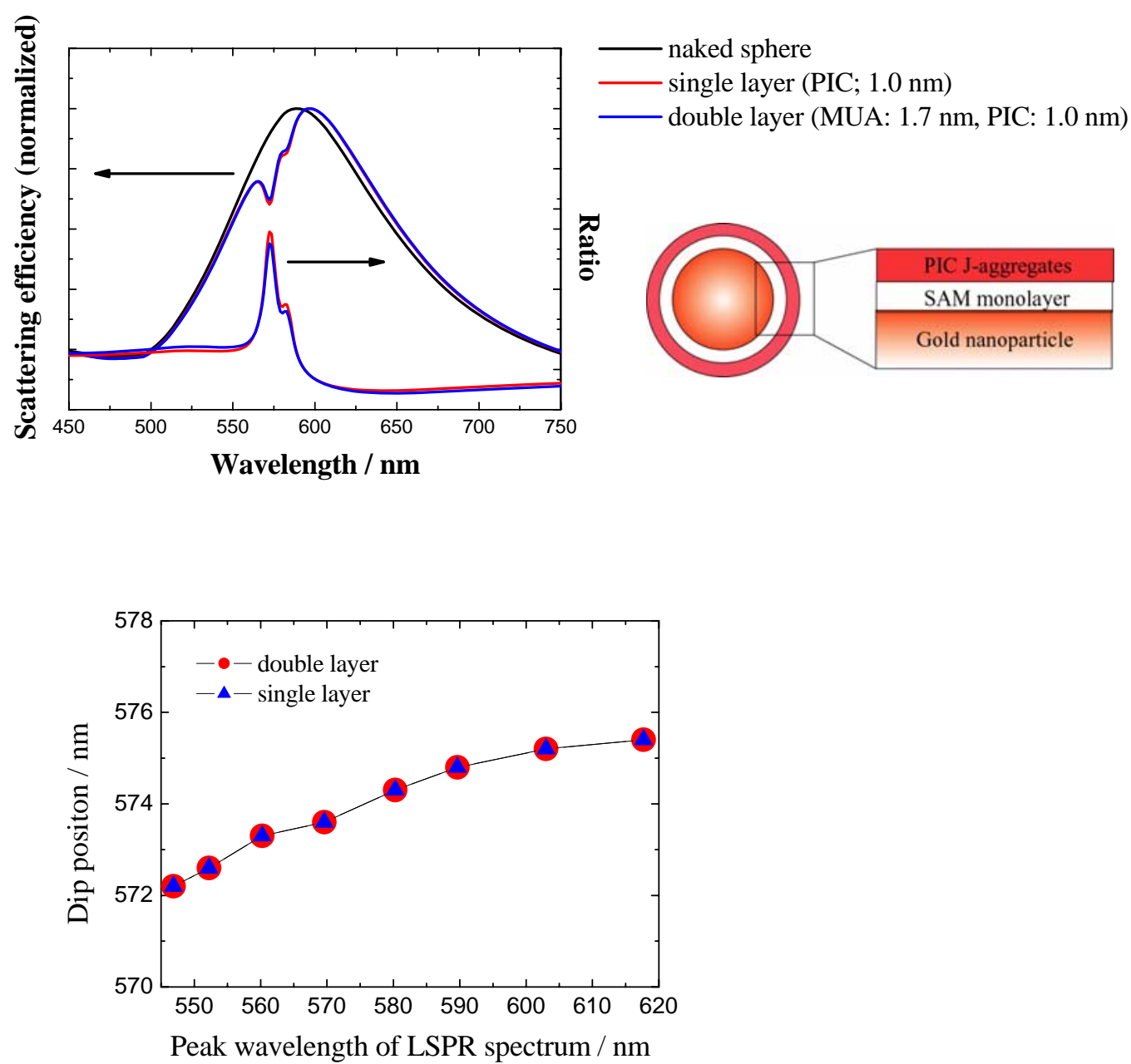

Figure S2. Numerical calculations for a double-layer coated model. (Top) Blue spectra show the calculated scattering spectrum of the gold core (100-nm diameter) coated with a spacer layer of MUA (1.7- nm thickness and the refractive index of 1.45) and the further coated with the PIC J-aggregate layer (1.0-nm thickness), and the spectral ratio (see text). Red spectra are the calculation of the core coated with the PIC J-aggregate layer. (Bottom) The calculated dependence of the dip position on the LSP resonance peak of the gold core for the core coated with single layer of the J-aggregate and with double-layer of the MUA SAM and the PIC J-aggregate. The effect of the spacer layer is negligibly small in the theoretical prediction based on the Mie theory. 